\title{
Un des $N$ et un $N$ en lecture partitive
}

\section{Anne Theissen}

\section{Abstract}

The constructions un des $N$ and $u n N$ enter in competition when the simple indefinite phrase un $N$ is employed partitively as the complex phrase un des $\mathrm{N}$. Our contribution aims to resolve the questions that put this parallel. In a way more precise, we will try to measure the semantic and syntactic resemblances and differences of the two partitive formulas, to evoke certain of their employment constraints and to put in value the originality of the partitive way borrowed by each one.

\section{Citer ce document / Cite this document :}

Theissen Anne. Un des $N$ et un $N$ en lecture partitive. In: Langages, $37^{\mathrm{e}}$ année, $\mathrm{n}^{\circ} 151,2003$. Indéfinis, définis et expression de la partition. pp. 67-86;

doi : https://doi.org/10.3406/lgge.2003.924

https://www.persee.fr/doc/lgge_0458-726x_2003_num_37_151_924

Fichier pdf généré le 03/05/2018 
Anne THEISSEN

Université Marc Bloch de Strasbourg E EA 1339 L.DL-Scolia

\section{UN DES N ET UN N EN LECTURE PARTITIVE 1}

\section{Introduction}

La particularité des déterminants indéfinis tels que $\| n$, de’s, ou dés indéfinis comme quelque's, certains, plusieurs, aucun, chaque, trois, etc., mais aussi la grande difficulté inhérente à leur analyse réside notamment dans la possibilité qu'ils ont de fonctionner en lecture partitive/forte et en lecture existentielle/ faible ${ }^{2}$. Si l'on considère des couples comme un/un des $N$, quelque's $N /$ quelques-uns des $N$, certains $N$ /certains des $N$, plusieurs/plusieurs de's $N$, aucun/aucun de's $N$, chaque/chacun de's $N$, trois / trois des $N$, etc., le second membre du binôme correspond à une structure partitive prépositionnelle clairement attestée (cf. Milner, 1978). Mais on sait également aujourd'hui, le fait a été souvent noté, que le fonctionnement du premier élément est plus complexe. En effet, si le second membre de ces couples implique nécessairement une lecture partitive, le premier (cf. un $N$, cortains $N$, plusicurs $N$, etc.) peut lui tantôt fonctionner en emploi existentiel et tantôt en emploi partitif. C'est dire que la structure un de's $N$, par exemple, mais aussi la forme un $N$ sont des marqueurs de partition. En dépit de cette (re)connaissance fonctionnelle (cf. Milner, 1978), ces deux modes partitifs n'ont pas fait l'objet, à notre connaissance du moins, d'une étude comparative. Pourtant la problématique ne peut manquer d'attirer l'attention de par son empan méthodologique d'une part - elle concerne l'ensemble des indéfinis -, de par ses vertus explicatives, d'autre part. La compréhension de cette situation devrait en effet non seulement éclairer le fonctionnement sémantico-référentiel des indéfinis mais aussi apporter un éclairage inédit sur le marquage et l'expression de la partition.

Pour aborder cette question, nous partirons d'une double hypothèse. Il y a ressemblance entre les deux structures en vertu de leur fonctionnement partitif. Un ou des point(s) commun(s) entre les deux formules réside(nt) nécessairement dans leur appariement à un ensemble de départ duquel est extrait un ou des individu(s) désigné(s) par les SN indéfinis partitifs. Mais il y a aussi divergence, comme le souligne Kleiber (2001a), lorsqu'il rappelle que

1. Un grand merci à Ignace!

2. I'our une revue de la littérature et la description des lectures existentielle et partitive, cf. notamment Kleiber (20)1a). 
si l'on dispose d'un ensemble de départ spécifique sur lequel on extrait les référents dénotés par des $\mathrm{SN}$ comme un $N$, certains $N$, quelque's $N$, etc., leur interprétation peut être équivalente à celle de un des $N$, quelques-uns, certains des, aucun des, etc., mais ne s'y assimile pas.

Nous essaierons ici de mesurer les ressemblances et les différences sémantiques et syntaxiques des deux formules partitives, d'évoquer certaines de leurs contraintes d'emplois et de mettre en valeur l'originalité du chemin partitif emprunté par chacune. Nous traiterons cette problématique à travers un cas particulier, celui de l'article indéfini $u n$. Pour ce faire, nous proposerons d'abord une description sémantico-référentielle comparative des formes $u n$ des $N$ et $u n N$. À partir des hypothèses posées, nous examinerons ensuite les situations où un des $N$ ne peut remplacer $u n N$ et vice-versa sans provoquer une autre interprétation du SN indéfini originel (existentielle vs partitive). Enfin, il s'agira d'expliquer le choix discursif entre les deux expressions partitives, c'est-à-dire le pourquoi de l'emploi du tour partitif un des $N^{3}$ ou celui de la forme partitive un $N$ lorsque les deux formules sont à même de marquer la partition.

\section{Un des $\mathbf{N}$ vs un $N$ : structure syntaxique et structure sémantique}

\subsection{La forme un des $\mathrm{N}$}

Les formes un des $N$, un de ces $N$, un de ses $N$ ou déterminants complexes (Bosveld-De Smet, $2000: 25)$ présentent cinq propriétés :

(i) il n'y a pas de nom-tête après un

(ii) le $\mathrm{N}$ tête absent ne peut être interprété que comme celui du SN du SP 4

(iii) le SN du SP est défini

(iv) le SN du SP est au pluriel

(v) le SN du SP ne peut être générique ${ }^{5}$

Pour ce qui est de (i) et (ii) cela revient à dire que le SN un des arbres de (1): Un des arbres a été abattu.

\footnotetext{
3. Il serait évidemment intéressant de prendre en compte le fonctionnement partitif de l'un des $N$. l'our des raisons de clarté et de place nous n'en ferons pas état ici. Voir Schnedecker (1998, 2000, $2001)$ pour le rapport entre un et l'un et la valeur de l'article défini dans ces structures.

4. A supposer, évidemment, que la suite de' + dét. $+N$ est un syntagme prépositionnel, ce qui peut être discute.

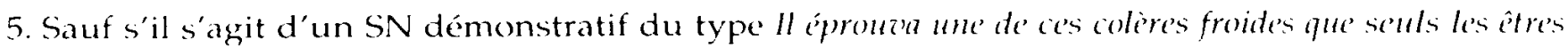
supréricurs peutent ressentir.
} 
équivaut à un arbre de + les arbres ou à un arbre des arbres (Milner, 1978) ${ }^{\circ}$. Le nom-tête absent arbre correspond donc au $N$ qui apparaît dans le SN subséquent. Aussi, quel que soit le statut syntaxique, pronom ou déterminant, que l'on donne à $u n$ dans la structure $u n$ des $N$, l'absence du nom-tête différencie fondamentalement le tour partitif un des $N$ de la forme $u n N$ où, comme on le sait, le nom-tête est généralement nécessaire $\left({ }^{*} U n\right.$ a été $\left.a b a t t u\right)$. Elle confère iconiquement à l'article indéfini $u n$ une certaine autonomie par rapport à la catégorie $\mathrm{N}$, puisque dans ce tour, il ne nécessite pas sa mention explicite. Cette autonomie ou cette absence du nom-tête permet de poser une première hypothèse - sur laquelle nous reviendrons - qui est que l'indéfini $u n$ ou le pronom renvoie à une occurrence qui n'est pas explicitement ou directement identifiée ou catégorisée comme $\mathrm{N}$.

La propriété (iii) se manifeste par l'impossibilité d'avoir un déterminant "indéfini " pluriel comme de's, cinq (cardinal), quelque's, plusieurs, etc. :

(2) *Un d'arbres / * Un de cinq/quelques/plusieurs arbres a été abattu.

Le trait (iv), à savoir la pluralité de l'ensemble, s'explique par l'impossibilité d'extraire un élément d'un singleton. Et cet ensemble d'extraction est différent de celui que suppose la mention singulière, mais toutefois intrinsèquement plurielle d'un terme collectif comme le montre *un de la forêt pour désigner un arbre de la forêt. Ce qui corrobore également l'idée que le $N$ absent doit être du même type que le $\mathrm{N}$ du syntagme d'extraction.

Le trait (v) marque que le SN défini du SI dés $N$ ne peut pas correspondre au SN générique le's $N$. Un dés arbrés ne peut signifier ou désigner une occurrence de la classe générique arbré. Trois raisons à cela. La première provient de la valeur sémantique de $N$. Un nom N, comme arbre, fournit la classe des arbres, c'est-à-dire que le $N$ arbre présuppose l'existence de la classe générique des arbres. Aussi, et c'est là la seconde raison, si l'on souhaite extraire un élément de cette classe, il n'est nullement nécessaire de construire formellement cet ensemble - donc de passer par la structure un des $N$ - puisque le nom à lui seul présuppose et donc fournit déjà la classe désirée. On comprend alors que, et c'est une troisième façon d'expliquer que le $\mathrm{SN}$ dans un des $N$ est restreint, si on opte pour le tour un des $N$, la classe ainsi construite - pour éviter tout phénomène tautologique - est une autre classe que celle des $\mathrm{N}$ génériques, puisque celle-ci est déjà donnée par le nom seul ${ }^{7}$.

6. Pour une autre analyse, voir Kupferman (1999).

7. Fn théorie, rien n'empêche de penser que la structure un de's $N$ ne puisse opérer sur un ensemble restreint générique avec une interprétation de sous-classe ou taxinomique. Cependant des exemples du type: Un des arbres, le saule pleureur, donne beancoup d'ombre; Un de's chats, le siamois, est iraiment tris beau, nous paraissent peu naturels. 
La caractérisation (i)-(v) nous permet d'aborder celle de la partition opérée. Quatre traits se dégagent :

(a) l'existence d'un contraste interne à l'ensemble de départ

Le tour partitif un des $N$, parce qu'il exprime explicitement le prélèvement d'un élément de l'ensemble d'extraction, a pour conséquence d'opposer l'élément extrait aux autres éléments de l'ensemble présupposé ou, si l'on préfère, d'opposer l'élément qui vérifie le prédicat à ceux qui ne le vérifient pas (cf. Kleiber 2001a). Ainsi (3):

(3) Les policiers inspectèrent la voiture. Une des roues était pleine de boue (Kleiber, 2001a)

suppose par défaut que toutes les roues, ou plus précisément les autres roues de la voiture, ne sont pas pleines de boue. En effet, si toutes les roues étaient pleines de boue, on ne voit pas pour quelles raisons on extrairait une roue de cet ensemble. L'extraction via la prédication qui accompagne le tour partitif se comprend donc comme un moyen d'opposer l'individu ainsi saisi aux autres individus de l'ensemble de départ. Partant, il y a un contraste interne à l'ensemble de départ qui oppose l'occurrence extraite à laquelle est attribuée une propriété - ici 'être pleine de boue' - et les autres éléments de l'ensemble de départ qui ne partagent pas cette propriété.

(b) une identification en termes d'appartenance

Un deuxième résultat lié au mode fonctionnel de la partition - c'est l'hypothèse que nous ferons - est que l'entité extraite n'est pas catégorisée directement comme N. Elle ne l'est qu'indirectement par l'intermédiaire de son appartenance à l'ensemble restreint des roues en (3), par exemple ${ }^{8}$. Certes il s'agit d'une roue, mais il s'agit en quelque sorte d'abord ou avant tout d'une roue de l'ensemble restreint \{roues\} rendu disponible par le SN défini la voiture. Nous sommes maintenant à même de répondre à la question posée plus haut, qui était de savoir comment se fait l'identification de l'occurrence dans la structure un des $N$. Si on accepte que le $N$ catégoriel est absent et donc qu'il n'intervient qu'indirectement ou en second lieu, alors on peut avancer l'hypothèse que l'identification se fait par rapport à l'ensemble d'extraction. Un argument en faveur de cette thèse est que, comme nous l'avons souligné, l'indéfini un dans un des $N$ a acquis une certaine indépendance par rapport à la catégorie, alors que le lien qu'il entretient avec l'ensemble d'extraction est plus contraint, comme le prouve son impossible suppression :

(4) Les policiers inspectèrent la voiture. ${ }^{*}$ Une était pleine de boue.

8. A rapprocher du fait que "le rapport de l'un à la tête nominale n'est pas "direct", comme il l'est dans le cas des anaphores nominales, mais il se trouve, en quelque sorte, médiatisé par l'ensemble, défini, auquel appartient l'entité qu'il désigne " (Schnedecker, 1998: 184). 
Le $\mathrm{N}$ catégoriel apparaît donc moins essentiel que l'ensemble d'extraction dans la forme un des $N$, comme le montre l'absence du premier et la présence obligatoire du second. Aussi, avec le tour partitif un des $N$, ce n'est pas la catégorie $\mathrm{N}$ qui est mise en avant, en termes d'identification occurrentielle, mais l'appartenance à l'ensemble d'extraction : l'individu n'est catégorisé comme $N$ qu'indirectement, c'est-à-dire en tant qu'élément d'un ensemble restreint de N. Et cette identification plutôt ensembliste que catégorielle entraîne le contraste interne que nous avons souligné. L'élément extrait étant saisi comme un élément d'un ensemble restreint, il rend saillant ou manifeste le contraste entre lui et les autres éléments de cet ensemble. Aussi peut-on poser l'hypothèse que, si la mise en saillance de ce contraste est pertinente dans le site discursif où se présente le tour partitif, cette structure sera un bon candidat pour exprimer la partition.

(c) l'ensemble de départ : le garant de la spécificité des occurrences partitives

La forme un des $N$ se caractérise par une autre propriété fonctionnelle : la spécificité des SN partitifs est garantie par l'ensemble de départ ou l'ensemble d'extraction (cf. Kleiber, 2001a). En effet, la spécificité de l'occurrence désignée par le tour partitif ne provient pas ou pas uniquement du prédicat, puisque les référents partitifs sont présentés comme des éléments d'un ensemble déjà installé, connu ou accessible. La lecture partitive (cf. Kleiber, 2001a) ne nécessite donc pas un prédicat spécifiant, puisque la spécificité est acquise par l'appartenance à l'ensemble présupposé représenté par le SN défini. On comprend alors la possibilité de (5) et le peu de naturel de (6) :

(5) Un des arbres est vraiment petit.

(6) ? Un arbre est petit.

En (5) l'ensemble d'extraction garantit la spécificité de l'occurrence désignée alors qu'en (6), la lecture existentielle ne peut se réaliser adéquatement parce que précisément la spécificité de l'occurrence, comme on le rappellera ultérieurement, est acquise par le biais du prédicat.

(d) l'occurrence un des $N$ : du connu et du nouveau à la fois

Enfin, comme dernier point, l'on peut opposer, d'une certaine façon, selon la dichotomie connu/nouveau, l'occurrence partitive et l'ensemble d'extraction. Si on accepte le caractère présupposé de l'ensemble d'extraction qui est une classe restreinte de $N$, on peut lui opposer l'occurrence individuelle extraite en la qualifiant d'entité posée ou en quelque sorte nouvelle dans le discours. En réalité, les choses sont un peu plus complexes. En effet, l'entité désignée par le tour partitif est à la fois connue et nouvelle. Elle est connue de par son statut d'élément de l'ensemble de départ, mais elle est aussi nouvelle dans la mesure où elle est extraite de cet ensemble. De là découle l'idée que la nouveauté liée à la forme un des $N$ équivaut à la mise en saillance ou en focus de l'élément $\mathrm{N}$ extrait. 


\subsection{La forme Un N}

La forme $u n N$ se distingue, en première analyse, du tour partitif un des $N$ par la nécessité du $\mathrm{N}$ au côté de l'indéfini et l'absence d'un ensemble d'extraction explicite. Aussi, contrairement à un des $N$ où l'identification se fait prioritairement par l'appartenance à l'ensemble d'extraction, l'entité désignée par un $N$ partitif est, elle, explicitement catégorisée comme N. L'identification est catégorielle d'abord, l'entité est considérée comme étant du type de $\mathrm{N}$, soit la catégorie donnée par le $\mathrm{N}$ du SN indéfini un $\mathrm{N}$. De là découle logiquement que la catégorie $\mathrm{N}$ bénéficie d'une saillance plus grande que l'ensemble d'extraction qui, lui, n'est qu'indirectement donné. Enfin, une troisième façon d'opposer la lecture partitive de $u n$ des $N$ et celle de $u n N$ consiste à postuler deux niveaux de contrastes différents. Dans le cas de un des $N$, nous avons signalé l'existence d'un contraste interne à l'ensemble de départ, c'est-à-dire une opposition entre l'élément extrait de cet ensemble qui vérifie le prédicat et les autres éléments de l'ensemble d'extraction qui ne le vérifient pas. Avec un $N$, il semble que l'identification catégorielle et la saillance dont bénéficie la catégorie $\mathrm{N}$ peuvent être accompagnées d'un contraste que nous qualifierons d'externe dans la mesure où il y a opposition catégorielle entre le $\mathrm{N}$ du SN indéfini et d'autres catégories $\mathrm{N}$.

Pour poursuivre la distinction entre $u n N$ et $u n \operatorname{des} N$, examinons l'information sémantique d'un SN du type un N. Pour Kleiber (2001a : 87), «il n'y a pas d'autres informations présentes communes que celles apportées par le déterminant indéfini et le nom, donc un sens de 'quantité de' par le déterminant et d'une quantité "partitive" de $\mathrm{N}$ obtenue par la combinaison avec le $\mathrm{N}$ qui suppose une catégorie ou une classe générique ». Les SN indéfinis sont donc sous-déterminés sémantiquement, comme le signale Corblin (1989), c'est-àdire que un $N$ a juste pour sens une occurrence de $N$ ou encore, comme le précise Kleiber (2001a: 88), "la quantité dénotée par le SN indéfini ne se trouve d'aucune manière distinguée d'autres quantités identiques : un homme, c'est un homme et c'est tout ». De là découlent trois conséquences :

-1-avec un $N$, contrairement à un des $N$, l'indéfini donne simplement l'entité comme une occurrence d'une catégorie, il n'y a donc pas d'indication que cette entité se trouve prise dans un ensemble restreint et/ou spécifique ;

-2- ce qui signifie que la lecture partitive de $u n N$, le cas échéant, n'est pas le résultat d'une configuration syntaxique comme c'est le cas avec un des $N$. Aussi, si un $\mathrm{N}$ donne lieu à un emploi partitif, cette lecture est nécessairement contextuelle ;

-3- il en va de même pour l'interprétation existentielle, entièrement dépendante, on le rappellera, du prédicat.

Revenons sur la caractérisation de "quantité partitive de $\mathrm{N}$ » (cf. Kleiber plus haut). Un $N$, même en lecture existentielle, suppose une opération partitive. En 
effet, la littérature sur les indéfinis " caractérise souvent non seulement les emplois partitifs des indéfinis mais aussi leurs emplois existentiels par une opération de prélèvement ou d'extraction. Ainsi pour (7) et (8):

(7) J'ai acheté un disque (Martin, 1983a : 174).

(8) Tu te souviens, le jour où j'ai vu un lion (Corblin, $1987: 42)^{\text {"1". }}$

certains parlent de partition au sens où est extrait de la catégorie générique ou virtuelle $N$ un élément (un lion ou un disque) qui se distingue des autres entités possibles, puisque précisément il n'y a que l'unité désignée par le SN indéfini qui se voit distinguée par le prédicat et par là même identifiée. Mais, comme le souligne Milner (1978:67), "alors que les tours partitifs consistent à circonscrire un sous-ensemble dans un ensemble déterminé, les tours quantitatifs circonscrivent un ensemble indéterminé, c'est-à-dire en fait la classe maximale dénotée par le nom " ". Et cette différence entre la lecture existentielle et la lecture partitive à proprement parler se manifeste clairement par l'absence de tout effet partitif en emploi existentiel. En effet, même si en toute logique rien ne l'interdit, rien ne donne à penser que le je de (7) n'a pas acheté les autres disques ou que le je de (8) n'a pas vu d'autres lions ce jour-là. Si l'on conçoit fort bien en quoi il est possible de parler de prélèvement ou de partition en emploi existentiel, il reste à comprendre l'absence d'effet partitif. P'our Kleiber (2001a), cette absence tient (i) à la nature de l'ensemble d'extraction et (ii) au rôle du prédicat. Il illustre son propos à partir de (9) et (10) :

(9) Un avion s'est écrasé hier dans les Vosgges.

(10) Une roue a été arrachée.

L'occurrence un arion en (9) est extraite de la classe générique $\mathrm{N}$ indéterminée avion. En (10), une rou' désigne une occurrence extraite d'un ensemble déterminé, restreint et spécifique, \{les roues de la voiture\}. Aussi, le rôle du prédicat n'est-il pas du tout le même dans les deux cas. En (10), la spécificité de $u n N$ n'est pas garantie par le prédicat mais par l'ensemble d'extraction, puisque ce dernier est déjà spécifique. Aussi, même si le prédicat distingue une roue particulière, la roue ainsi distinguée, mais aussi les autres roues de l'ensemble, ont déjà acquis la spécificité par leur appartenance à l'ensemble d'extraction. Le rôle du prédicat se limite en quelque sorte à individualiser en termes événementiels l'entité désignée par un $N$ : la roue dont on parle est celle qui a été arrachée. Et s'il y a effet de partition c'est parce qu'on peut supposer que les autres éléments de l'ensemble, eux aussi spécifiques, on le rappelle, ne présentent pas cette propriété, sinon le prédicat ne jouerait pas son rôle de spécifieur. Il y a donc une scission au sein de l'ensemble entre les éléments qui vérifient le prédicat et ceux qui ne le vérifient pas.

9. Kleiber (2001a) cite à cet égard Culioli (1977), Martin (1983 a et b), Corblin (1987, 1989).

10. Exemples cités par Kleiber (2001a : 77).

11. La citation est reprise à Kleiber (2001a : 78). 
Or cette division et l'effet partitif qui en résulte n'ont pas cours en emploi existentiel. En effet, le rôle du prédicat en (9) est autre parce qu'il s'applique à un élément extrait de la classe générique, c'est-à-dire à une entité $N$ qui n'est pas spécifique au départ. Le rôle du prédicat consiste donc avant tout à porter à l'existence un élément $\mathrm{N}$ : soit un avion qui est spécifique, parce que, précisément, il s'est écrasé hier dans les Vosges. Dans ce cas, n'est donc spécifique que l'élément qui vérifie le prédicat. Par conséquent, il ne peut y avoir opposition entre l'élément qui vérifie le prédicat et ceux qui ne le vérifient pas, ces derniers n'étant nullement portés à l'existence.

\subsection{Bilan et perspectives}

La lecture partitive de $u n$ des $N$ et celle de $u n N$ s'opposent en tout premier lieu sur un point essentiel : avec un des $N$ l'interprétation est directement donnée par le sens de la construction syntaxique alors que, avec un $N$, elle est acquise par d'autres facteurs lexicaux et/ou contextuels.

Outre ce point fondamental, d'autres éléments opposent la lecture partitive de ces deux structures:

- du côté de un des $N$

(i) l'absence d'un nom tête au côté de un dans la structure un des $N$ confère à un une certaine indépendance vis-à-vis de $\mathrm{N}$. Un tel constat nous a conduite à poser comme première hypothèse que l'article-pronom dans un des $N$ renvoie à une occurrence qui n'est pas explicitement ou directement identifiée ou catégorisée comme étant $\mathrm{N}$;

(ii) le mode opératoire partitif de $u n$ des $N$, c'est-à-dire le fait que un des $N$ exprime explicitement le prélèvement d'un élément dans un ensemble d'extraction, permet de postuler :

- une opposition interne à l'ensemble d'extraction : l'élément extrait est opposé aux autres éléments de l'ensemble présupposé ainsi que semble en témoigner la question lequel plus licite après cette configuration que qui ça ?: J'ai rencontré un des profs de la fac. - Lequel ? / Qui ça?

- une identification ensembliste ou en termes d'appartenance: l'occurrence extraite de l'ensemble de départ est avant tout catégorisée par son appartenance à l'ensemble d'extraction; elle n'est donc identifiée comme $\mathrm{N}$ qu'indirectement, par le biais de son appartenance à un ensemble de $\mathrm{N}$;

- la mise en saillance ou tout au moins une importance plus grande accordée

à l'ensemble d'extraction qu'à la catégorie $\mathrm{N}$ elle-même. Le fait que l'on ait de + les exprimé après $u n$ entraîne, cognitivement, la mise en saillance de l'ensemble défini SN. La trace du mode de donation de la spécificité de un du $N$ par l'ensemble d'extraction se trouve dans la nature définie du complément partitif de $u n$. 
- du côté de $u n N$ :

(i) l'occurrence désignée par un $N$ est explicitement catégorisée comme $\mathrm{N}$ : l'identification est donc catégorielle, d'abord, l'entité étant considérée comme étant du type de $\mathrm{N}$, soit la catégorie donnée par le $\mathrm{N}$ du SN indéfini un $N$ comme le corrobore la meilleure adéquation de la question qui ça? par rapport à l'emploi partitif de un prof de la fac: J'ai rencontré un prof de la fac. - Quiça? / Lequel?

(ii) la catégorie $\mathrm{N}$ bénéficie d'une saillance ou d'une importance plus grandes que l'ensemble d'extraction qui, lui, n'est qu'indirectement donné ;

(iii) l'identification catégorielle peut être accompagnée d'un contraste externe, c'est-à-dire d'une opposition catégorielle entre le $\mathrm{N}$ du SN indéfini et d'autres catégories $\mathrm{N}$.

L'on peut certes prévoir des cas où l'une ou l'autre de ces structures peuvent convenir, c'est-à-dire des situations où la différence d'interprétation est minime. Mais nous ferons aussi l'hypothèse que, si les deux structures présentent une lecture partitive dans un même site, le choix de l'une ou l'autre est lié au mode de présentation occurrentielle proposé et à l'intégration adéquate du mode partitif choisi dans la stratégie informationnelle en cours. Avant d'étudier ces situations de concurrence partitive, nous ferons un rapide point sur les situations où la substitution d'une structure à l'autre est difficile ou conduit vers une lecture différente.

\section{Situations de non-concurrence}

La description fonctionnelle et sémantique que nous avons donnée du tour partitif $u n$ de's $N$ permet de mieux saisir la différence majeure entre $u n N$ et $\| n$ dés $N$, à savoir l'impossibilité d'une lecture existentielle pour $u n$ dés $N$, alors que cette lecture correspond à une des lectures les plus fréquentes de un $N$. Cette différence se manifeste de deux façons lorsqu'on substitue à $u n N$ existentiel le tour partitif $u n$ des $N$ : soit l'emploi de $u n$ des $N$ est maladroit, voire difficile, soit l'emploi de un des $N$ est possible, parce que le contexte autorise la construction d'un ensemble de $N$ qui peut servir de base extractive, mais la lecture existentielle disparaît alors au profit d'une lecture partitive.

\subsection{Un des $\mathrm{N}$ : un emploi impossible}

Dans les exemples (11) et (12),

(11) En feu sur l'autoroute. Une voiture (? une des voitures) a pris feu sur l'A4 hier matin vers $8 \mathrm{~h} 30$, à la hauteur de la sortie de Bischeim. (DNA, novembre 2001) 
(12) Après une soirée très arrosée en compagnie de deux amis, Dalziel se retrouve impliqué dans un accident de voiture. Un cycliste (? un des cyclistes) est mort. (TV magazine, février 2002)

l'absence d'un ensemble de $\mathrm{N}^{12}$ conduit nécessairement vers la lecture existentielle de un $N$ et rend caduc l'emploi du tour partitif. La lecture existentielle de un $N$, on l'a vu, suppose que la spécificité de l'occurrence désignée est donnée par le prédicat et non pas par un ensemble de départ de $\mathrm{N}$ duquel cet individu serait extrait. Et assurément, il ne peut en être autrement en (11) et (12), puisque précisément il n'y a pas d'ensemble de départ disponible. Aussi l'absence d'un tel ensemble implique-t-elle nécessairement que la construction de la spécificité de l'individu un $N$ se réalise d'une autre façon. Et c'est là que le prédicat joue son rôle de spécifieur. Le fonctionnement respectif de la lecture existentielle et du tour partitif expliquent non seulement pourquoi le tour partitif ne peut pas fonctionner - l'absence d'un ensemble de N, c'est-àdire d'un ensemble d'extraction - mais aussi en quoi la lecture existentielle est adéquate. Le prédicat portant à l'existence l'occurrence un $N$, la lecture existentielle ne nécessite nullement la présence d'un quelconque ensemble de départ ${ }^{13}$. Cela apparaît également d'une façon tout à fait révélatrice avec (13) et (14) où il y a un ensemble d'extraction disponible, mais qui n'est pas un ensemble de $\mathrm{N}$ :

(13) Ma tante pouvait tremper dans l'infusion bouillante dont elle savourait le goût de feuille morte ou de fleur fanée une petite madeleine dont elle me tendait un morceau quand il était suffisamment amolli (? un des morceaux quand il était suffisamment amolli). (Du côté de chez Swann, M. Proust, 24)

(14) Mayotte: policiers en grève. Une partie des agents de la police territoriale de Mayotte (? Une des parties des agents de la police territoriale de Mayotte) est en grève illimitée. Ils souhaitent la revalorisation de leur salaire de base, (...) (DNA, décembre 2001)

Ces exemples présentent deux points communs : (a) la présence d'une entité qui peut être assimilée à un tout ou à un ensemble d'extraction (cf. une petite madeleine et les agents de la police territoriale de Mayotte) et (b) l'usage d'un $\mathrm{N}$ méronymique ${ }^{1+}$ (cf. morceau et partie). Pourtant la lecture partitive de $u n$ des $N$ reste maladroite. La présence d'un tout déterminé ou défini n'est en effet pas suffisante. La lecture partitive nécessite un ensemble d'éléments individués ou délimités qui sont des N. Or, en (13) et (14), le tout n'est pas présenté comme découpé par avance en morceaux et en parties que le SN indéfini pourrait désigner ou l'article indéfini extraire. Le SN indéfini ne peut donc pas saisir une partie dans un ensemble d'éléments, parce que, précisément, de

12. I. absence d'un ensemble d'extraction se révèle notamment par l'incongruité de questionne-

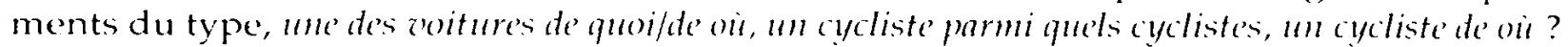

13. On rappelle ici, comme l'a montré Kleiber $(1981,2001$ a), la nécésisité d'un prédicat spécifiant.

14. Le sens lexical des $N$ méronymiques peut favoriser une anaphore associative indéfinie, soit la lecture partitive de $m$ N (cf. Kleiber, 20()1a et b). 
telles parties ne préexistent pas. Il n'y a de parties que celles désignées par le SN indéfini qui les porte à l'existence. L'adéquation de la lecture existentielle et l'absence d'effet partitif s'expliquent alors. On ne peut en effet, par défaut d'occurrences $\mathrm{N}$ disponibles déjà délimitées, faire la différence entre une partie qui vérifie le prédicat et d'autres qui ne le vérifieraient pas. Ainsi, la madeleine de (13) n'est pas découpée par avance en morceaux, de même le fait de signaler qu'une partie des agents de la police territoriale de Mayotte est en grève n'implique pas que cette police est déjà prédivisée en parties. La partie désignée par un $N$ est construite par le prédicat et non pas antérieurement à l'événement que ce dernier décrit. Voilà pourquoi la lecture existentielle s'impose ici : elle amène à l'existence la partie désignée par un $N$, alors que la lecture partitive s'appuierait sur l'existence préalable des parties en distinguant la partie qui vérifie le prédicat de celles qui ne le vérifient pas, différence qu'illustrent également (15) et (16) :

(15) Une des parties de l'immeuble a été repeinte.

(16) Une partie de l'immeuble a été repeinte.

En (15), l'immeuble est déjà distribué en 'parties' (le premier étage, la cave, etc.), et il y a celle qui est repeinte et celles qui ne le sont pas. Et cet énoncé ne peut signifier que seule la moitié de la cage d'escalier, par exemple, a été repeinte alors que (16) peut convenir dans un tel cas. Le SN unce partic de l'immeleble peut en effet désigner n'importe quelle partie, même la moitié de la cage d'escalier, au sens où la partie désignée n'est pas antérieure au fait de la repeindre, puisque c'est précisément le prédicat - l'action 'repeindre' - qui construit, délimite ou porte à l'existence la partie. Mais (16) peut aussi se lire en termes véritablement partitifs. Le SN indéfini désigne alors une des parties déjà disponibles, qui est spécifique de par son appartenance à l'ensemble d'extraction (cf. le premier étage, la cave, etc.) comme peut le faire un morcén du vasé en (17):

(17) Le vase s'est cassé en mille morceaux. Un morceau/un des morceaux s'est glissé sous le canapé.

où le SN un morceau aussi bien que un de's morceaux s'interprètent en termes partitifs, même si, on le verra, le chemin partitif ou la présentation partitive est différent(e).

\subsection{Un des $\mathrm{N}$ : un emploi possible}

Un $N$ existentiel peut accepter à sa place un des $N$ lorsque le contexte autorise la construction d'un ensemble d'extraction :

(18) Trafic au stade. Un ancien stade de Varsovie (un des anciens stades de Varsovie) est devenu le centre de la contrefaçon en Europe de l'Est. (DNA, novembre 2001)

(19) Braquage à la bombe lacrymogène. Une boulangerie de la route de Schirmeck (une des boulangeries de la route de Schirmeck) a été victime hier vers $6 \mathrm{~h}$ d'un braquage. (DNA, novembre 2001) 
En (18) et (19), les informations données par N, la présence d'une entité définie et les connaissances stéréotypiques que l'on a des entités dénotées alliées à l'emploi de l'extracteur de autorisent la construction d'un ensemble de départ de $N$ sur lequel la partition est rendue possible. Elle intervient lorsque l'on souhaite indiquer qu'il y a plusieurs $N$ concernés et lorsqu'il s'agit de signaler que tous les membres de cet ensemble $N$ ne vérifient pas le prédicat. Cette interprétation apporte alors une information que ne peut fournir univoquement la lecture existentielle de $u n N$. On le vérifiera en faisant l'opération inverse, c'est-à-dire en substituant en (20) à un des $N$ le SN un N :

(20) Une centaine de caravanes se sont installées hier après-midi sur un des parkings relais tram de Hoenheim (un parking relais tram de Hoenheim). Un parking de la CTS reste disponible pour les automobilistes. (DNA, novembre 2001)

Avec un des $N$, un lecteur - notamment non alsacien - accepte, sans doute et sans en avoir eu connaissance auparavant, l'existence de plusieurs parkings relais à Hoenheim. Le tour partitif fournit ainsi, par son mode opératoire, une information que ne peut évidemment livrer la forme existentielle un N. Le référent, en lecture existentielle, n'étant pas appréhendé comme élément d'un ensemble, les autres éléments ne sont pas non plus évoqués parce que précisément il $n^{\prime} y$ a pas d'ensemble. La forme un des $N$, si le site lui est favorable, impose donc une stratégie informationnelle particulière. Il y a une restructuration informationnelle qui conduit à la construction d'un ensemble de départ qui s'impose tout naturellement à l'interprétant qui, de fait, accepte en même temps, s'il ne la connaît pas encore, l'existence d'autres $N$ qui, eux, ne vérifient pas le prédicat.

En (20), on vient de le voir, le remplacement du tour partitif par un $N$ provoque la disparition de la lecture partitive au profit d'une lecture existentielle spécifique. En (21), il amène une lecture existentielle non spécifique comme le met en évidence l'ajout de l'expression n'importe laquelle révélatrice de l'interprétation non spécifique ( $c f$. Lyons, $1978: 154$ ) :

(21) Mais quand paraissait un peu épuisé le pouvoir qu'avait de le faire souffrir un des mots prononcés par Odette (un mot prononcé par Odette, n'importe lequel), alors un de ceux sur lesquels l'esprit de Swann s'était moins arrêté jusque-là, un mot presque nouveau venait relayer les autres et le frappait avec une vigueur intacte. (Du côté de chez Stwann, M. Proust, 167)

L'interprétation de un $N$ se distingue ici de celle que modélise le tour un des $N$ pour deux raisons au moins. La première tient évidemment au fait que le SN indéfini un $N$ change de cap interprétatif, puisque d'une lecture partitive instanciée par un des $N$ l'on passe à une lecture existentielle. La seconde raison - plus méconnue celle-ci - réside dans l'abandon d'une interprétation spécifique au profit d'une interprétation non spécifique. En effet, alors que $u n$ des $N$ s'accompagne d'une interprétation spécifique, un $N$ conduit plutôt vers la non-spécificité. Le tour partitif un des $N$ remplit donc ici une double fonction : il établit l'interprétation partitive et - fonction plus méconnue - il stabilise la 
lecture spécifique. On comprend comment se réalise cette seconde fonction si on tient compte du statut et du rôle qui incombent à l'ensemble d'extraction. En lecture partitive, on le rappelle, l'ensemble d'extraction équivaut à un ensemble défini et restreint qui garantit la spécificité à ses éléments. Aussi, contrairement à un $N$ existentiel où la spécificité de l'occurrence est fournie par le prédicat, l'occurrence extraite par le tour partitif est nécessairement spécifique de par son appartenance à un ensemble de départ spécifique et donc indépendamment du prédicat.

\section{Interprétations partitives de un $\mathrm{N}$ et un $\operatorname{des} \mathrm{N}$}

À côté des cas de proximité comme (22):

(22) Pour faire partie du "petit noyau ", du "petit groupe ", du "petit clan " des Verdurin, une condition était suffisante mais elle était nécessaire, il fallait adhérer tacitement à un Credo dont un des articles (un article) était que lé jeune pianiste, protégé par Mme Verdurin cette année-là et dont elle disait : "Ça ne devrait pas être permis de savoir jouer Wagner comme ça ! (..) (Du côté dé chez Sưann, M. Proust, 86)

il est des cas où le choix de $u n N$ ou celui de $u n$ de's $N$ entraîne des différences dans la manière de présenter le référent. Pour étudier les différentes possibilités, nous partirons des situations où la structure $u n \mathrm{~N}$ a été choisie.

La focalisation sur l'appartenance de l'occurrence à la catégorie $N$ se prête à différentes nuancés de sens ou peut être plus ou moins différemment "récupérée " par le discours. Pour des raisons de clarté, nous distinguons ici différentes situations, mais plusieurs facteurs peuvent en réalité concourir simultanément pour justifier le choix de $m$ N. Quoi qu'il en soit, toutes les situations présentent un point commun : le discours fournit, d'une manière ou d'une autre, un ensemble d'extraction fini ou une catégorie discursivement restreinte à l'image de l'ensemble défini sur lequel opère le tour partitif un de's $N^{15}$.

\subsection{Catégorie $\mathrm{N}$ et ensemble d'extraction : le problème de la mise en saillance}

Un exemple comme (23) illustre la prévalence de la catégorie N sur l'ensemble d'extraction :

(23) Et justement le premier dîner chez les Verdurin auquel assista Forcheville mit en lumière toutes ces différences, fit ressortir ses qualités et précipita la disgrâce de Swann.

15. Il serait évidemment intéressant d'étudier comment le discours construit une sous-catégorie ou une sous-classe de $N$ spécifique pour ne pas risquer de tomber du cóté de la lecture existentielle avec un changement de róle pour le prédicat et donc une absence d'effet partitif. Mais le constat nous suffira ici. Voir à ce sujet Galmiche (1986). 
Il y avait à ce dîner, en dehors des habitués, un professeur de la Sorbonne (un des professeurs de la Sorbonne), Brichot, qui avait rencontré $M$. et Mme Verdurin aux eaux et, si ses fonctions universitaires et ses travaux d'érudition n'avaient pas rendus très rares ses moments de liberté, serait volontiers venu souvent chez eux.

Car il avait cette curiosité, cette superstition de la vie qui, unie à un certain scepticisme relatif à l'objet de leurs études, donne dans n'importe quelle profession, à certains hommes intelligents, médecins qui ne croient pas à la médecine, professeurs de lycée qui ne croient pas au thème latin, la réputation d'esprits larges, brillants, et même supérieurs. (Du côté de chez Szuann, M. Proust, 114)

Le choix de un $N$ s'explique par le mode de présentation du référent. L'individu est, par l'emploi de cette tournure, directement placé dans la catégorie $\mathrm{N}$ professeur de la Sorbonne. Il est ainsi présenté comme professeur de la Sorbonne sans passer par un ensemble d'extraction. Avec un des $N$, au contraire, l'individu est saisi par rapport aux autres éléments de l'ensemble d'extraction, c'est-à-dire en opposition aux autres professeurs de la Sorbonne. Et c'est donc l'ensemble des professeurs de cette université qui se trouve focalisé. Or ce passage met en avant les qualités des convives et leur appartenance à un corps de métier particulier. Il est donc plus pertinent de focaliser l'appartenance de l'individu à la catégorie professeur de la Sorbonne, en opposition éventuelle avec d'autres corps de métier (cf. médecin, professeur de lycée), que de présenter ce nouveau convive en opposition aux autres professeurs de la Sorbonne, c'est-à-dire de l'appréhender comme élément de l'ensemble d'extraction les professeurs de la Sorbonne.

Le choix de un $N$ et celui de la mise en saillance de la catégorie $N$ qui en résulte peuvent aussi s'expliquer par une autre différence d'interprétation. En effet, lorsqu'un adjectif est antéposé à $N$, l'information adjectivale participe différemment à la lecture partitive selon la structure partitive choisie :

À Rockwell, une petite ville des États-Unis (une des petites villes des États-Unis), en octobre 1957, un petit garçon regarde la télévision seul chez. lui. Brusquement, l'image se brouille. (TV magazine, 2001)

(...) le nom d'AXA n'y (= TF1) a jamais été cité. Parce que c'est un gros annonceur de la chaîne (un des gros annonceurs de la chaîne). (LC canard enchainci, novembre 2001)

La partition opère dans des ensembles d'extraction différents selon la portée de l'adjectif. Dans le cas de un des $N$, la partition se réalise dans les ensembles "les petites villes des États-Unis", "les gros annonceurs de la chaîne". L'occurrence ainsi désignée appartient à une sous-catégorie "les petites villes des États-Unis", par exemple, et s'oppose en tant que telle à d'autres éléments de cet ensemble, soit d'autres petites villes des Etats-Unis. Le choix de un $N$ propose une identification différente. La lecture partitive un $N$ évolue dans les ensembles "villes des États Unis", "annonceurs de la chaîne ". Il s'agit donc d'une ville des États-Unis qui a la propriété d'être 
petite. En termes d'opposition, cette ville peut s'opposer à d'autres villes qui, elles, ne seraient pas petites, par exemple. La portée de l'adjectif est donc différente selon que l'information qu'il apporte participe ou non à la construction de l'ensemble d'extraction. Par là même, nous semble-t-il, le choix de la structure un $N$ confère plus de poids informationnel à la propriété donnée par l'adjectif. Cette dernière, n'étant pas partagée par tous les éléments de l'ensemble d'extraction, permet de distinguer l'occurrence extraite de celles qui ne le sont pas. La structure un $N$ met donc en avant l'appartenance à la catégorie $N$ de l'entité et la qualité qu'elle a d'être petite alors que le tour partitif un des $N$ penche, comme on va le voir plus manifestement avec les exemples ci-dessous, vers une identification plus individualisante.

\subsection{Une identification catégorielle opposée à une identification plus individualisante}

Le choix de un $N$ peut aussi marquer l'indifférence quant à une identification occurrentielle plus précise :

(26) (...) la tradition voulait qu'on eût donné à Assuérus les traits d'un roi de France (...) (Du coté de chez Sieam, M. Proust, 27)

(27) Un jour, ayant rencontré dans un livre de Bergotte (...) une plaisanterie que le magnificue et solennel langage de l'écrivain rendait encore plus ironique (...) (Du côté de chez Szermn, M. Proust, 44)

L'emploi de un $N$ se comprend d'abord en termes de saillance catégorielle. Mais ce choix stratégique peut s'accompagner d'une autre volonté, peut-être même d'une forme d'indifférence. En (26) et (27), il s'agit évidemment d'un roman spécifique ou d'un roi de France précis. Or, le fait de choisir une identification avant tout ou surtout catégorielle nous éloigne d'une identification plus particulière comme celle proposée par $u n$ de's $N$ en (28):

(28) Et ainsi - tandis que quelque artiste qui, lisant les Mémoires du XvII" siècle et désirant de se rapprocher du grand Roi, croit marcher dans cette voie en se fabriquant une généalogie qui le fait descendre d'une famille historique ou en entretenant une correspondance avec un des souverains actuels de l'Europe (un souverain actuels de l'Europe), tourne précisément le dos à ce qu'il a le tort de chercher sous des formes identiques et par conséquent mortes - (...) (Du coté de chez Swann, M. Proust, 54)

L'emploi de un des $N$ en (28), contrairement à celui de $u n N$, implique une focalisation sur l'ensemble d'extraction. L'identification de l'occurrence, parce qu'elle est ensembliste, est plus précise. L'élément est en effet présenté en opposition à d'autres éléments du même type $\mathrm{N}$ comme en témoigne la meilleure adéquation ici de la question avec lequel que avec qui ?

Outre la focalisation de l'ensemble d'extraction, l'emploi de un des $N$ présente un autre intérêt : elle conduit vers une identification plus individualisante. Le statut pronominal de $u n$ dans cette structure dirige en effet vers 
une identification plus particulière. Aussi, si l'on souhaite aller vers une individuation plus grande ou accentuer l'importance de l'identité occurrentielle, la structure un des $N$ est adéquate comme l'illustrent (29) où un des collègues se distingue des autres collègues par une certaine promesse et (30) où l'identité de l'individu - bien plus que le statut d'élève que mettrait en avant l'emploi de un $N$ - est importante par rapport au drame narré :

(29) Tenez, justement le mari de l'amie chez qui je vais en ce moment (ce qui me donne le très grand plaisir de faire route avec vous) lui a promis, s'il est nommé à l'Académie ( $c^{\prime}$ est un des collègues du docteur / c'est un collègue du docteur) de lui faire faire son portrait par Machard. (Du côté de chez Swann, M. Proust, 170)

(30) Alors qu'il aide à améliorer la sécurité d'un collège, Walker ne parvient pas empêcher le suicide d'un des élèves (d'un élève). (TV Magazine, mars 2002)

À l'inverse, si une identification en tant que membre de la catégorie $\mathrm{N}$ est jugée suffisante, la forme un $N$ l'emporte :

(31) Sur une île du Pacifique (une des îles du Pacifique), deux jeunes filles sont retrouvées mortes sur le rivage. (TV Magazine, mars 2002)

(32) Un mystérieux terroriste fait exploser une bombe dans un magasin de New York (un des magasins de New York) et menace de mettre la ville à feu et à sang. (TV Magazine, avril 2002)

L'identification de l'occurrence en tant que $\mathrm{N}$ suffit dans les contextes (31) et (32). On ne souhaite en effet pas mieux identifier l'île particulière ni connaître plus précisément le magasin piégé. Il s'agit simplement de présenter ces deux endroits comme étant d'un certain type N. Il n'y a pas non plus d'intérêt à identifier ces occurrences par rapport à d'autres ou à les opposer aux autres îles du Pacifique ou aux autres magasins de New-York. Le fonctionnement partitif de un des $N$ et un $N$ expliquent aussi les divergences d'interprétations entre (33) et (34) :

(33) Je voudrai avoir écrit un article de Rebielk.

(34) Je voudrai avoir écrit un des articles de Rebielk.

En (33) un article de Rebielk se paraphrase par " peu importe lequel, pourvu que ce soit un article de Rebielk" comme le montre la pertinence de la question quoi? alors que (34) penche vers une identification plus individualisante et légitime la question lequel?. On comprend également pourquoi (35) paraît moins naturel que (36):

(35) Je voudrai avoir écrit un article de Rebielk et Paul les autres.

(36) Je voudrai avoir écrit un des articles de Rebielk et Paul les autres.

si on accepte que un $N$ propose une identification plutôt catégorielle alors que un des $N$ penche vers une identification plus individualisante (cf. l'article portant sur la disparition de la licorne dans les Vosges). Cette différence de saisie et donc de présentation occurrentielle est également confirmée par des sites où un de's $N$ s'avère plutôt maladroit : 
(37) (..) où un reflet de jour avait pourtant trouvé moyen de faire passer ses ailes jaunes, et restait immobile entre le bois et le vitrage, dans un coin (? un des coins), comme un papillon posé. (Du côté de chez Swann, M. Proust, 38)

(38) Une fois la porte fermée, il souleva un coin du store (? un des coins du store) et observa si, dans la cour, les lampes des voisins étaient toutes éteintes. (Le livre d'un seul homme, Gao Xingjian, 95)

Il est en effet superfétatoire ici de distinguer plus précisément l'identité de l'occurrence désignée ou de la saisir en opposition aux autres éléments de l'ensemble pas plus qu'il n'est pertinent de mettre en avant l'ensemble d'extraction. L'on n'a pas, nous semble-t-il, par exemple, l'idée de s'enquérir des autres coins par une question du type et les autres (coins)? Le syntagme prépositionnel dans un coin en (37) localise un individu par rapport à un autre type d'endroit et non pas par rapport à un autre coin de la pièce. De même, il est peu naturel de souligner pour (38) que les autres coins du store ne vérifient pas le prédicat, c'est-à-dire n'ont pas été soulevés, ou encore de s'interroger plus avant sur une identité plus précise de l'occurrence. L'essentiel est de présenter le référent comme un élément de la catégorie $N$ plutôt qu'un élément d'un ensemble de $N$ fini, même si cette information n'est pas ignorée. Or, si l'identification est d'abord catégorielle, la tournure partitive un $N$ est plus adéquate. L'exemple (39) en témoigne également :

(39) Passe moi un sucre (? un des sucres).

Si l'on imagine que le destinataire a devant lui un sucrier rempli de sucres, on a donc bien un ensemble de $N$, soit un ensemble de sucres. Or, là aussi, l'emploi du tour partitif $u n$ des $N$ est inapproprié. En effet, peu importe aux protagonistes que l'occurrence désignée par un de's $N$ appartienne à un ensemble de $N$, l'essentiel réside en son appartenance à la catégorie $N$ sucre. La catégorie $N$ est ici prioritaire par rapport à l'ensemble restreint auquel l'occurrence appartient. De plus, a priori, les éléments 'sucre' sont identiques. C'est dire que le locuteur ne souhaite pas un élément précis en opposition aux autres éléments de l'ensemble, il souhaite simplement un élément de la catégorie N (n'importe lequel). La désignation et l'identification de l'occurrence se contentent de l'information «être $N$ " ${ }^{16}$.

\subsection{Contraste externe vs contraste interne}

Le choix de un $N$ s'explique aussi par la nature du contraste que cette tournure partitive peut installer :

(40) Quelquefois, comme Éve naquit d'une côte d'Adam (une des côtes d'Adam), une femme naissait pendant mon sommeil d'une fausse position de ma cuisse. Formée du plaisir que j'étais sur le point de goûter, je

16. C'est pour cette raison que l'on a, à côté de léa saigne à une dé sés lèrres, Léa saignè à une lèrre et non *Léa saigné à une de's lère's (note du lecteur anonyme que je remercie). 
m'imaginais que c'était elle qui me l'offrait. (Du côté de chéz Szann, M. Proust, 2)

(41) Mais j'avais revu tantôt l'une, tantôt l'autre, des chambres que j'avais habitées dans ma vie, et je finissais par me les rappeler toutes dans les longues rêveries qui suivaient mon réveil ; chambres d'hiver où quand on est couché, on se blottit la tête dans un nid qu'on se tresse avec les choses les plus disparates : un coin de l'oreiller (un des coins de l'oreiller), le haut des couvertures, un bout de châle, le bord du lit, et un numéro des Débats roses, qu'on finit par cimenter ensemble (...) (Du côté de chez Swann, M. Proust, 4)

Au vu des descriptions antérieures, l'on s'explique la différence d'interprétation des SN partitifs une côte d'Adam et une des côtes d'Adam. Un des $N$ pointe vers une identification plus individualisante comme en témoigne la question laquelle alors que un $N$ ne pointe pas vers cette même question. Mais l'information essentielle réside dans l'appartenance de l'occurrence à la catégorie côte. Il y a donc virtuellement au moins une opposition, ce que nous avons nommé contraste externe, entre l'occurrence qui est de type côte et d'autres occurrences qui seraient d'un autre type de N. Or dans les exemples (40) et (41), ce contraste intègre adéquatement la stratégie informationnelle en cours. On peut ainsi noter l'opposition des $\mathrm{N}$ côte et cuisse en (40) et celle entre coin, haut, bout, bord en (41). Mais le contraste suscité par un $N$ peut aussi être simplement donné à ou suscité chez l'interprétant sans être directement ou lexicalement "récupéré » par le discours. Ainsi en est-il en (42) où un $N$ oppose le collège désigné à d'autres collèges d'Alsace et où le SN un élève de $4^{e} d u$ collège de Strasbourg inscrit en pointillé une opposition aux élèves de $3^{\circ}$ (cf. contraste externe), par exemple, alors que le SN un des élèves de $4^{\circ}(. .$. opposerait l'élève aux camarades de sa classe et le collège cité aux autres collèges de Strasbourg (contraste interne) :

Les parents d'un élève exclu d'un collège de Strasbourg (d'un des collèges de Strasbourg) ont déposé une requête en annulation de cette décision. Le commissaire du gouvernement a demandé son rejet.

Un élève de $4^{\mathrm{e}} \mathrm{du}$ collège de Strasbourg (un des élèves de $4^{\mathrm{e}} \mathrm{du}$ collège de Strasbourg) avait été définitivement exclu de l'établissement pour "absences injustifiées" suite à une décision du conseil de discipline confirmée par le recteur en août 1998. (DNA, novembre 2001)

L'emploi de un $N$ ou celui de un des $N$ s'interprète aussi comme l'adéquation d'un contraste externe, pour le premier, et d'un contraste interne, pour le second. Cette différence d'interprétation contrastive contribue ainsi à expliquer le choix et les nuances interprétatives de (43) et (44):

Un chien s'est échappé.

Un des chiens s'est échappé.

dans une situation (1) où se trouvent quatre chiens et trois chats et dans une situation (2) où l'on est en présence seulement de quatre chiens. En (1), aussi bien (43) que (44) peuvent convenir, mais avee des visées interprétatives différentes. 
Si l'on souhaite insister sur la nature du fuyard, $u n N$ est plus adéquat. La catégorie chien non seulement bénéficie d'une certaine saillance, mais surtout le contraste externe qui en résulte permet de servir pertinemment l'opposition chien/chat mise en avant dans cette interprétation. Si l'on s'intéresse davantage à l'identité particulière du fuyard, l'emploi de un dés chiens est plus adéquat, à la fois parce que cette tournure penche vers une individualisation plus grande et parce qu'elle suscite un contraste interne entre l'occurrence qui vérifie le prédicat et celles qui ne le vérifient pas. P'our ces mêmes raisons, un des $N$ est meilleur que un $N$ en (2). L'identification catégorielle en tant que chien étant déjà acquise dans ce cas et en raison de l'inadéquation d'un contraste externe, $u n \mathrm{~N}$ s'avère en effet maladroit en (2). À l'inverse, l'identification particulière et le contraste interne que met en avant un des $N$ servent adéquatement la situation.

\section{Conclusion}

Notre travail ne répond évidemment pas à toutes les questions que pose l'expression de la partition par les indéfinis. Quoi qu'il en soit, il nous semble que nos hypothèses éclairent sous un jour nouveau le fonctionnement sémantico-référentiel des indéfinis, apportent des réponses inédites à l'expression de la partition et proposent une explication cohérente, en termes discursifs, des options partitives que représentent le tour partitif un de's $N$ et la forme partitive un $N$. Nos hypothèses permettent en effet de distinguer le comportement partitif de ces deux types de structures et d'expliquer le choix de l'une ou l'autre de ces formules en tenant compte de l'originalité du chemin partitif tracé par chacune d'elles et les modes de présentations référentielles qui les caractérisent respectivement. 


\section{Bibliographie}

BOSVEI.D-de SMET L., 2000, Les syntagmes nominaux en de's et $d u$ : un couple curieux parmi les indéfinis, in Bosveld-De Smet L., Van Peteghem M., Van de Velde (éds), De l'indétermination à la qualification. Le's indéfinis, Artois, Université d'Artois, 17-116.

Culioli A., 1977, Notes sur Détermination et Quantification : définition des opérations d'extraction et de fléchage, Rapport ERA 642, Paris, Laboratoire de Linguistique formelle, I’aris, VII/CNRS.

CORBLIN F., 1985, Anaphore et interprétation des segments nominalix, Thèse de Doctorat d'État de l'Université de Paris VII.

CORBI.IN F., 1987, Indéfini, défini et démonstratif, Genève, Droz.

CORBI.IN F., 1989, Spécifique-générique : un modèle pour les indéfinis, Modèles linguistiques, $\mathrm{XI}, 2,11-35$.

GALMiCHE M., 1986, Référence indéfinie, événements, propriétés et pertinence, in David J. \& Kleiber G. (éds), Déterminants : syntaxé et sémantiqué, Paris, Klincksieck, 41-71.

KLEIBER G., 1981, Problemes de référence: Descriptions définies et nom propres, Paris, Klincksieck.

KLEIBER G., 2001a, Indéfinis : lecture existentielle et lecture partitive, in Kleiber G., Laca B., Tasmowki L. (éds), Typologie des groupes nominaux Rennes, Presses Universitaires de Rennes, 47-97.

KLEIBER G, 2001b, L'anaphore associative, Paris, PUF.

KUPFERMAN L., 1999, Réflexions sur la partition : les groupes nominaux partitifs et la relativisation, Langue française, 122, 30-51.

LyONS J., 1978, Éléments de sémantique, Paris, Larousse (trad. de Semantics, vol. 1, Cambridge, 1977).

Martin R., 1983a, Pour une logique du sens, Paris, PUF (2"éd., 1992).

MARTiN R., 1983b, De la double " extensité " du partitif, Langue française, 57, 33-43.

MILNLR J.C., 1978, Ordre et raisons de langue, Paris, Seuil.

SCHNEDECKER C., 1998, L'un et l'autre ou quelques aspects d'une union libre, Revtue de Sémantique et Pragmatique, 3 : 177-195.

SCHNEDH:CKER C., 2000, L'un...sans l'autre : encore un "indéfini » marginal, Scolia, 13, 195213.

SCHNEDECKER C., 2001, Couples anaphoriques et cohésion discursive : quand l'un/l'autre font bande à part, in De Mulder W., Co Vet., Vetters C. (éds), Anaphores pronominales et nominales. Études pragma-sémantiques, Amsterdam-New York, Rodopi. 